\title{
Post-traumatic hyperlipofuscinosis in the human retinal pigment epithelium
}

\author{
M K Ko, W R Lee, N M McKechnie, B Hall-Parker
}

\begin{abstract}
Light microscopy (including fluorescence microscopy) and electron microscopy were applied to a study of the photoreceptor-retinal pigment epithelium (RPE) complex in a human eye which had been severely traumatised nine months prior to enucleation. The main feature of interest was a massive accumulation of lipofuscin in the retinal pigment epithelium at the posterior pole, and quantitative fluorescence microscopy provided values three times those obtained in appropriate control tissue. The photoreceptor layer was normal at the posterior pole but became progressively atrophic towards the periphery. The concentration of lipopofuscin was proportional to the degree of preservation of the retinal photoreceptors. By electron microscopy the cells in the RPE were seen to be packed with a mixture of lipofuscin granules and melanolysosomal complexes, but occasional photoreceptor phagosomes were found. Bruch's membrane and the choriocapillaris were normal. We attribute this hitherto unreported abnormality of the RPE after trauma to a dysfunction consequent on an overload of the monolayer by photoreceptor debris at the time of trauma.
\end{abstract}

It is now accepted that there is constant renewal of photoreceptor outer segments and that groups of discs, bound within a membrane, are released from the photoreceptor tips and are phagocytosed and degraded by the cells of the retinal pigment epithelium (RPE). ${ }^{12}$ The biochemical processes which are involved in the enzymatic dissolution of the photoreceptor phospholipid are less well understood, but in the normal cell it seems likely that the end stage of a photoreceptor disc phagosome is a residual body. ${ }^{2}$ Morphological studies have shown that the number of lipofuscin granules increases with age in the RPE, particularly at the posterior pole,,$^{3-7}$ and the assumption is that this represents a dysfunction in the degradative mechanisms required for disc lipoprotein phagocytosis, though the autophagic destruction of mitochondrial membranes probably makes a contribution. ${ }^{46}$ Reports describing excessive amounts of lipofuscin in the RPE are rare and are limited, to a 'choroidal dystrophy', ${ }^{4}$ a case of retinitis pigmentosa, ${ }^{8}$ a carrier in chorioderaemia, ${ }^{9}$ and to ceroid lipofuscinosis in the human eye, ${ }^{10}$ and in animal tissues. ${ }^{11}$

It was therefore surprising to find a massive deposition of lipofuscin in the RPE in a traumatised eye which was submitted for routine examination. Since this complication of trauma has not to our knowledge been reported previously, we performed a morphological study to promote further understanding of the effects of overload of the RPE phagocytic systems by excessive photoreceptor disc material.

\section{Materials and methods}

\section{CLINICAL HISTORY}

A 55-year-old male was seriously injured in a fight in February 1982. He sustained a fractured skull and a fractured mandible, with severe contusion of the left orbit. A scleral rupture in the left eye was not noted until the orbital effusion had settled, and when first seen by an ophthalmologist the sclera had healed and intervention was not required. The patient failed to attend hospital until November 1982, when he returned complaining of severe pain. On examination the eye was disorganised and blind, and enucleation was performed on 24 November, some nine months after the injury. In the ensuing years the right eye has been normal.

\section{PATHOLOGY}

The globe was fixed in $4 \%$ glutaraldehyde in $0 \cdot 1 \mathrm{M}$ cacodylate buffer and measured $23 \times 22.5 \times 23$ $\mathrm{mm}$; the optic nerve was cut flush. The central part of the enucleated eye was embedded in paraffin wax and studied by serial section and conventional special stains which included periodic acid Schiff (PAS), Prussian blue, Bodian, and Loyez. Unstained sections were mounted in fluorescence-free mounting medium and examined in ultraviolet light (Leitz Pleomepi illumination system with excitation at $380-400$ $\mathrm{nm}$ and transmission at $460-480 \mathrm{~nm})$. For quantitative studies of the lipofuscin content of the pigment epithelium the UV fluorescence microscope was attached to an image analyser (Optomax System I; Micromeasurements Ltd, Cambridge). Control tissue from cases in which the RPE-photoreceptor complex was normal were used to obtain a baseline for comparison with the traumatised eye (Table 1), and for interest the analysis was also performed on an eye containing a metastatic tumour with partial exudative detachment and an eye which had siderosis bulbi (Table 1) to assess the respective 
Table 1 Area of lipofuscin $(\mu \mathrm{m})^{2}$ in a $50 \mu \mathrm{m}$ length of RPE

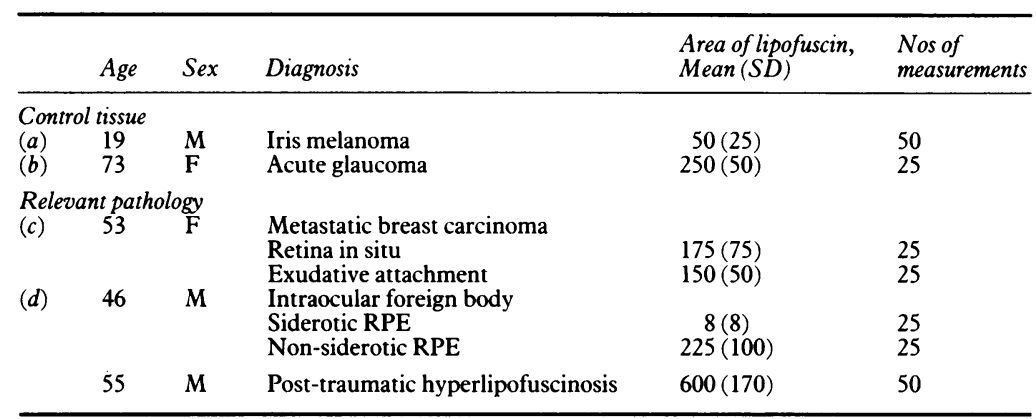

effects of retinal detachment and iron accumulation on the lipofuscin content. The sections were treated in an identical manner in terms of anatomical sampling for the measurement of lipofuscin. The measurements were expressed as the area of lipofuscin autofluorescence $(\mu \mathrm{m})^{2}$ per $50 \mu \mathrm{m}$ length of pigment epithelium.

Six blocks from the retina of the traumatised eye were postfixed with osmium tetroxide and processed through Araldite for electron microscopic study by a Philips 301 microscope. Two blocks from the regions of good photoreceptor preservation were not treated with osmium, so that lipofuscin and melanin granules in the RPE could be more easily differentiated.

\section{Results}

ROUTINE PATHOLOGICAL EXAMINATION

Macroscopic examination. The cornea was opaque and fibrotic, but transillumination revealed a superior iris defect, and the globe was opened in the vertical plane. The anterior chamber was narrowed by iridocorneal contact, and the globe was aphakic. Scar tissue replaced the superior parts of the iris and ciliary body, and vitreous traction folds were present at the retinal periphery; the posterior retina was in situ, but had a slate grey appearance. The mid periphery of the
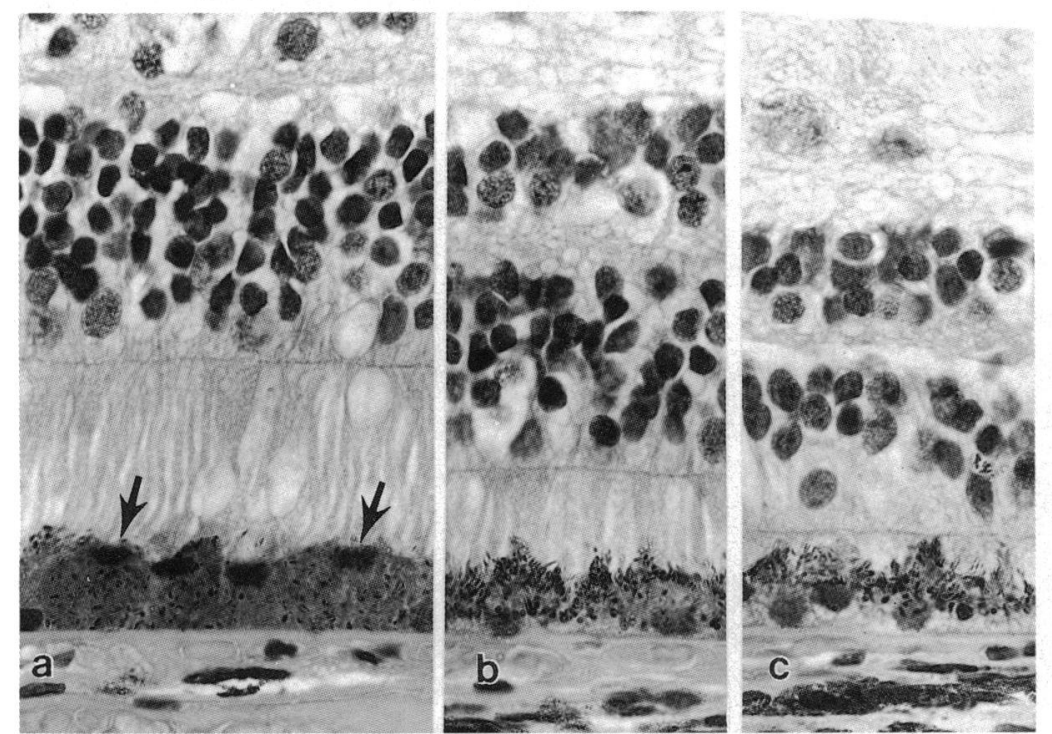

Figure 1 The light microscopic appearance of the outer retina at (a) the posterior pole, $(b)$ the mid periphery, and (c) toward the equator. Note the progressive atrophy of the photoreceptors in height and number and the loss of nuclei in the outer nuclear layer. In (a) the retinal pigment epithelial cells are enlarged by an accumulation of lipofuscin in the cytoplasm and there is internal displacement of some nuclei (arrows). Bruch's membrane and the choriocapillaris are normal. (Haematoxylin-eosin, $\times 475$.)
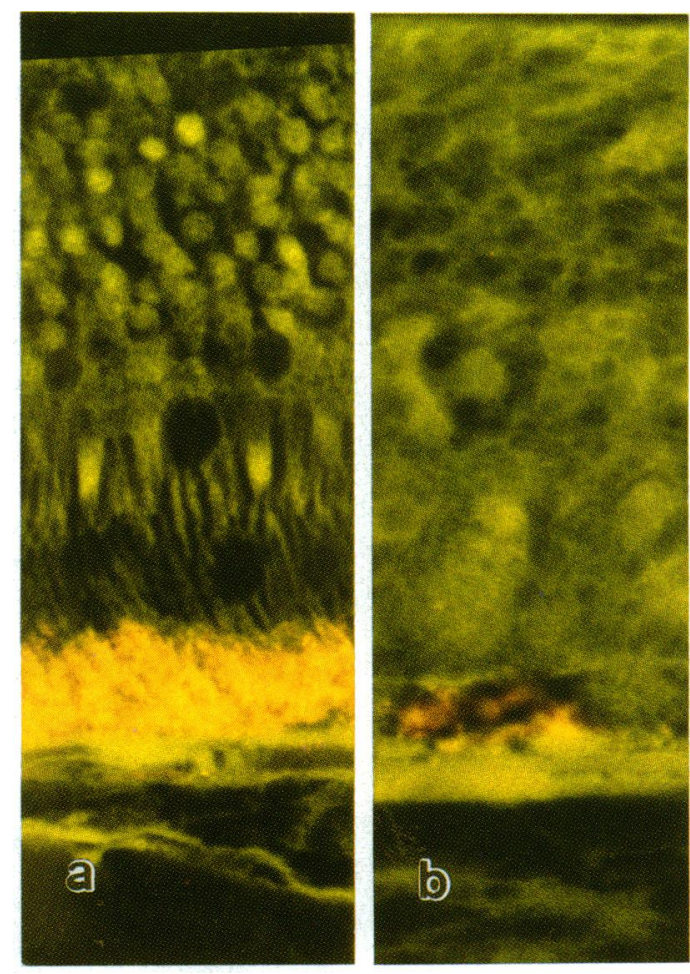

Figure 2 In ultraviolet light, the autofluorescence of lipofuscin granules is striking in the RPE at the posterior pole $(a)$, by comparison with the equator $(b) .(\times 630$.

retina showed a fine granular pigmentary disturbance with an almost linear series of small brown particles arranged in the coronal plane. A few hyalinised white vessels contained pigment granules in the walls, but the appearance was unlike that seen in post-traumatic pseudoretinitis pigmentosa. The macula was swollen by oedema, and the peripapillary retina was thickened by a small extrusion of myelin due to crushing of the optic nerve by a snare (toothpaste artefact).

Paraffin histology. The corneal pathology included stromal fibrosis, and the anterior segment was distorted by a fibrous ingrowth from the healed wound at the superior limbus; this ingrowth extended to the inferior iris leaf. Lens remnants were identified in the corneal wound, from which a fibrous band extended into the vitreous. This fibrous band contained haemomacrophages and had drawn the superior peripheral retina across the pars plana. Below, the inner leaf of a retinoschisis was drawn across the pars plana by vitreous traction. The uveal tract contained an inconspicuous lymphoplasmacytoid infiltrate.

Apart from a mild and sparse lymphocytic perivasculitis, the architecture of the inner twothirds of the retina was preserved. The following abnormalities were observed in the outer retina:

At the posterior pole there was cystoid macular oedema and a small exudative detachment beneath the fovea, but the perifoveolar photoreceptors were of normal architecture (Fig la). The RPE adjacent to the normal photoreceptors was swollen by accumulations of PASpositive granules, which in some cells displaced the nuclei to the ininer aspect (Fig la). The lipofuscin content was high (Fig 2a), and the iron stain was negative in the RPE in this region. 
Toward the midperiphery the inner and outer segments of the photoreceptors were shorter (Fig 1b). The lipofuscin content of the RPE cells was lower in this location.

Toward the equator the atrophy of the outer retina was more pronounced (Fig lc). At the equator the retinal pigment epithelium was flattened and atrophic, and clumps of pigment laden cells were present within the retina. The RPE and the cells within the retina stained positively for iron, but the ipofuscin content was markedly reduced in the RPE (Fig $2 b$ ).

QUANTITATIVE ANALYSIS OF LIPOFUSCIN CONTENT Values obtained for measurement of lipofuscin in the RPE in various specimens are shown in Table 1.

In the control specimens in which the posterior RPE and retina were of normal appear- ance there was a five-fold difference in the area of fluorescent material when values from the 19year-old (50, SD 25) and the 73-year-old (250, SD 50) were compared. There was considerable variation round the mean in the younger eye and a more uniform distribution in the older eye. The values obtained for lipofuscin in the RPE in the eye of a 53-year-old female with an isolated metastatic tumour were of a similar order irrespective of attachment (175, SD 75) or detachment (150, SD 50) of the retina. In the traumatised globe from a 46-year-old male in which intense iron deposition was observed in the RPE adjacent to an iron-containing foreign body, values for lipofuscin were remarkably low in the presence of iron $(8, S D 8)$, but were within the range provided by the other comparison specimens where iron staining was absent $(225$, SD 100). Values obtained for the lipofuscin content of the RPE at the posterior pole in the

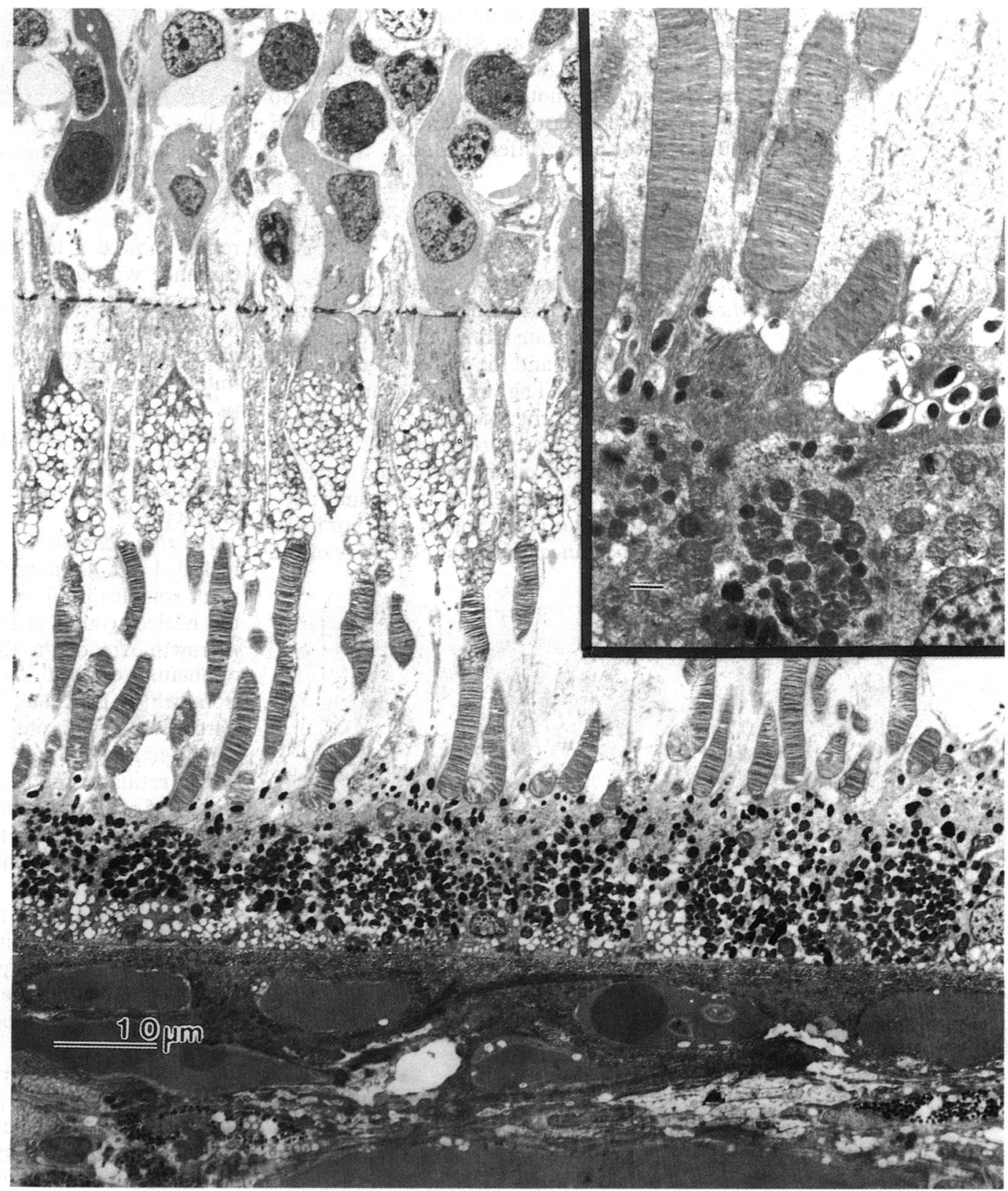

Figure 3 By electron microscopy the photoreceptors at the posterior pole are reduced in number but approach normal height. The $R P E$ cells are enlarged by numerous intracytoplasmic inclusions. Inset shows $R P E$ cell processes surrounding the tips of the photoreceptors. (Inset bar marker is $1 \mu \mathrm{m}$; osmicated tissue.) 
Figure 4 The majority of the intracytoplasmic granules represent various stages of lipofuscin degradation and fusion with the formation of complexes with melanin granules. Photoreceptor phagosomes are indicated by arrows. Note that the cell nucleus is displaced to the basal area. BM: Bruch's membrane. This tissue was not osmicated. (Bar marker is $1 \mu \mathrm{m}$.)

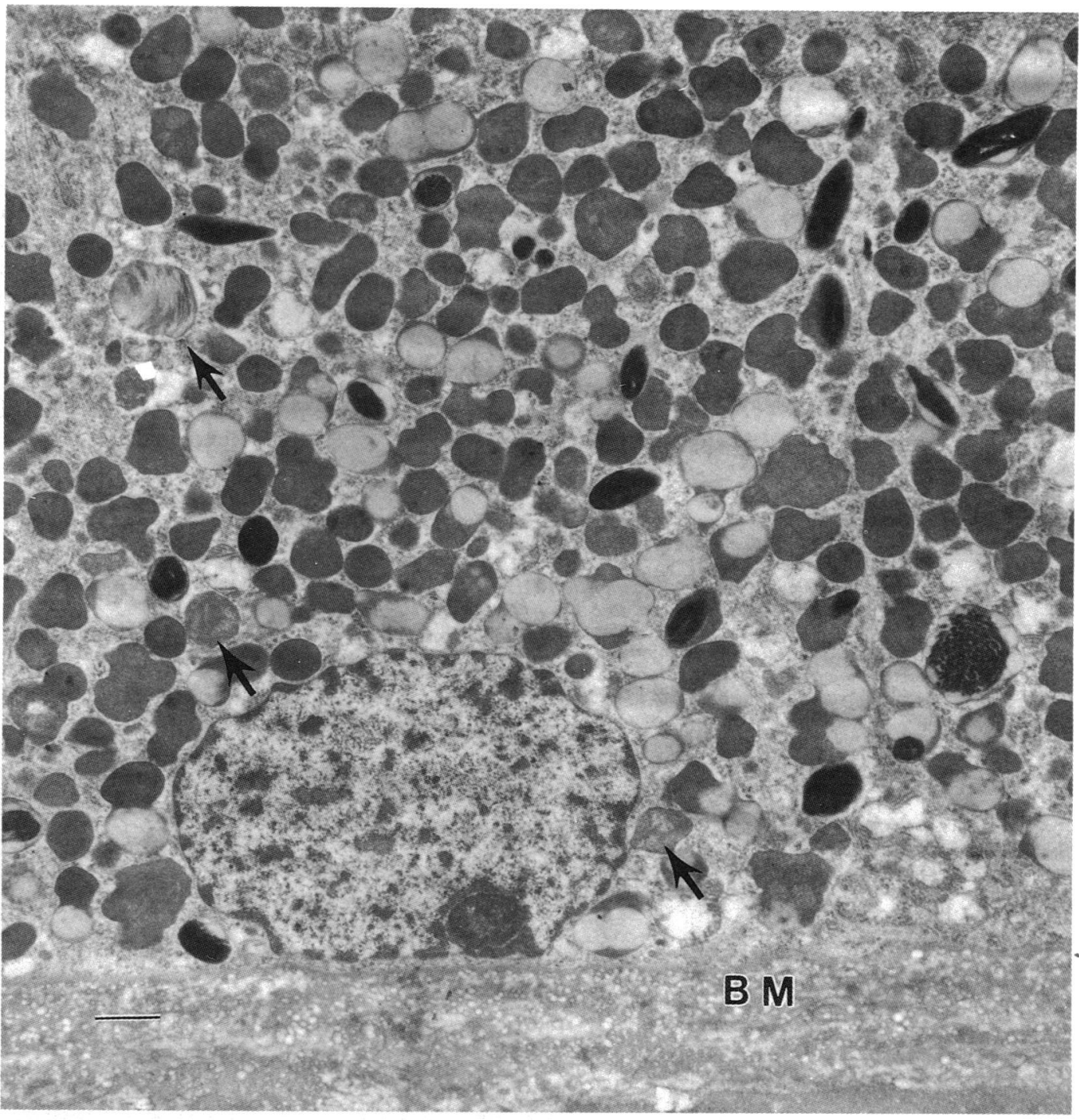

traumatised eye under investigation were at a very high level $(600, S D 170)$ by comparison with the other specimens.

\section{ELECTRON MICROSCOPY}

The ultrastructural preservation of the pigment epithelium and the photoreceptor outer segments was acceptable, though there was evidence of mitochondrial dissolution. The findings will be discussed in three sections related to the topography of outer retina. Notable pathological changes were not observed in Bruch's membrane or the choriocapillaris in any region.

\section{Regions where the outer segments approached normal size}

Where by light microscopy the parafoveal photoreceptor layer appeared to be normal, it was apparent by electron microscopy that there some reduction in the numbers of photoreceptors, and the interphotoreceptor matrix was expanded (Fig 3). The outer segments showed focal disc fragmentation. The cytoplasm of the RPE cells contained large numbers of electron lucent round or oval lipofuscin granules ( $1 \mu \mathrm{m}$ diameter), melanin-lipofuscin complexes, and some photoreceptor disc phagosomes (Figs 4 and
5). It was noteworthy that photoreceptor disc material was also identifiable in phagosomes in the basal part of the cells. The phagolysosomes were of irregular outline and contained packed $10 \mathrm{~nm}$ to $15 \mathrm{~nm}$ light and dark bands within a relatively homogeneous electron dense matrix (Figs 4 and 5). Melanosomes were found throughout the cytoplasm, and melanosome dissolution was evidenced by the formation of structures which contained beaded strands (striated melanoprotein) and a well defined peripheral electron lucent rim (Fig 7).

Regions in which photoreceptors were present but stunted

The obvious abnormality (Figs 1b, 1c, and 6) was shortening and distortion of the outer segments of the surviving cells. The inner segments were depleted and shrunken, and there was a reduction in nuclei in the outer nuclear layer. The tips of the photoreceptor outer segments appeared as isolated ovoid masses within elongated frond-like processes from the RPE cells, but occasionally were found within the apical RPE cytoplasm (Fig 6). The RPE monobodies, which had a more granular appearance than those at the posterior pole. Phagolysosomes layer contained excessive quantities of lipofuscin 

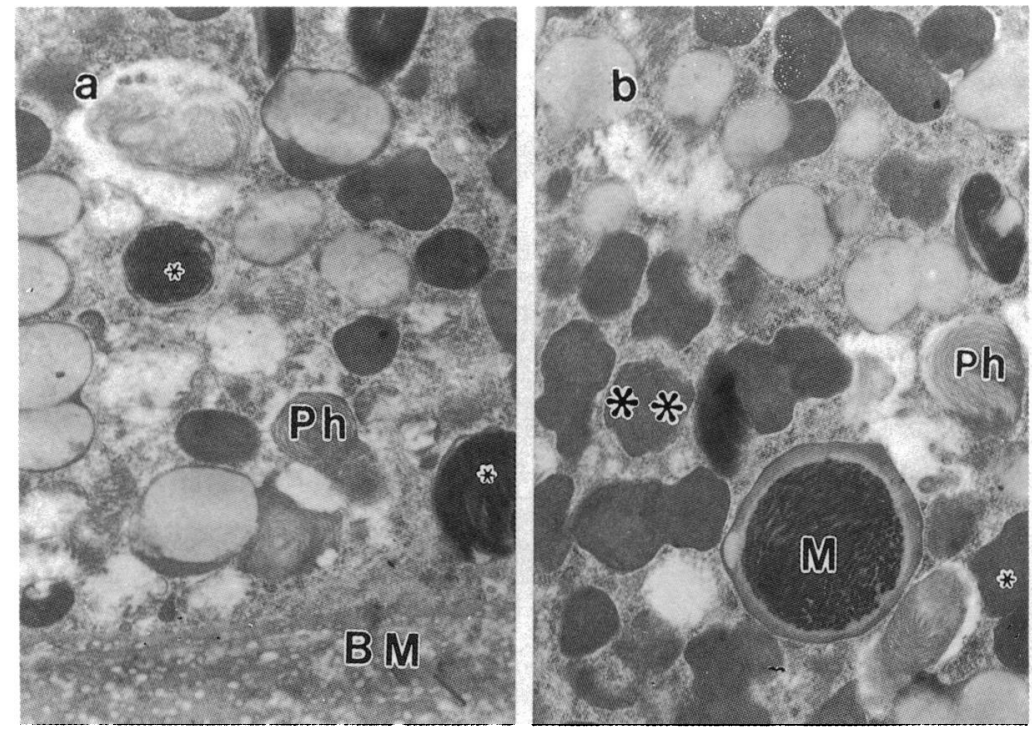

Figure 5 Details of the intracytoplasmic granules within the RPE. (a) Note the photoreceptor disc phagosomes (Ph) among the lipofuscin granules in the basal part of the RPE.BM:Bruch's membrane. In (b) occasional melanolysosomes $(M)$ have a large round profile containing striated melanoprotein. In $(a)$ and $(b)$ premelanolysosomes $(\star)$ have a more electron-dense content than prephagolysosomes $(\star \star)$. The relatively electronlucent structures are characteristic lipofuscin granules. This tissue was not osmicated. $(\times 12000$. $)$ and melanolysosomes were not found in the basal cytoplasm.

Regions of total photoreceptor cell atrophy were absent and replaced by glial cells (Fig 7). The processes from the glial cells interdigitated with those from the pigment epithelium, and in these regions some migrating RPE cells and some intraretinal macrophages were identified. The multilayered pigment epithelium in this region contained a few melanin granules and compound melanophagosomes, but lipofuscin granules were rare. The cells contained some electron dense granular material with a morphology consistent with that of breakdown products of blood. Macrophages containing similar electron dense particles and secondary melanophagolysosomes were found in the retina as solitary cells or in clumps.

\section{Discussion}

It is probably justifiable to explain the findings in
At the retinal periphery the photoreceptor cells

mo

7。

空

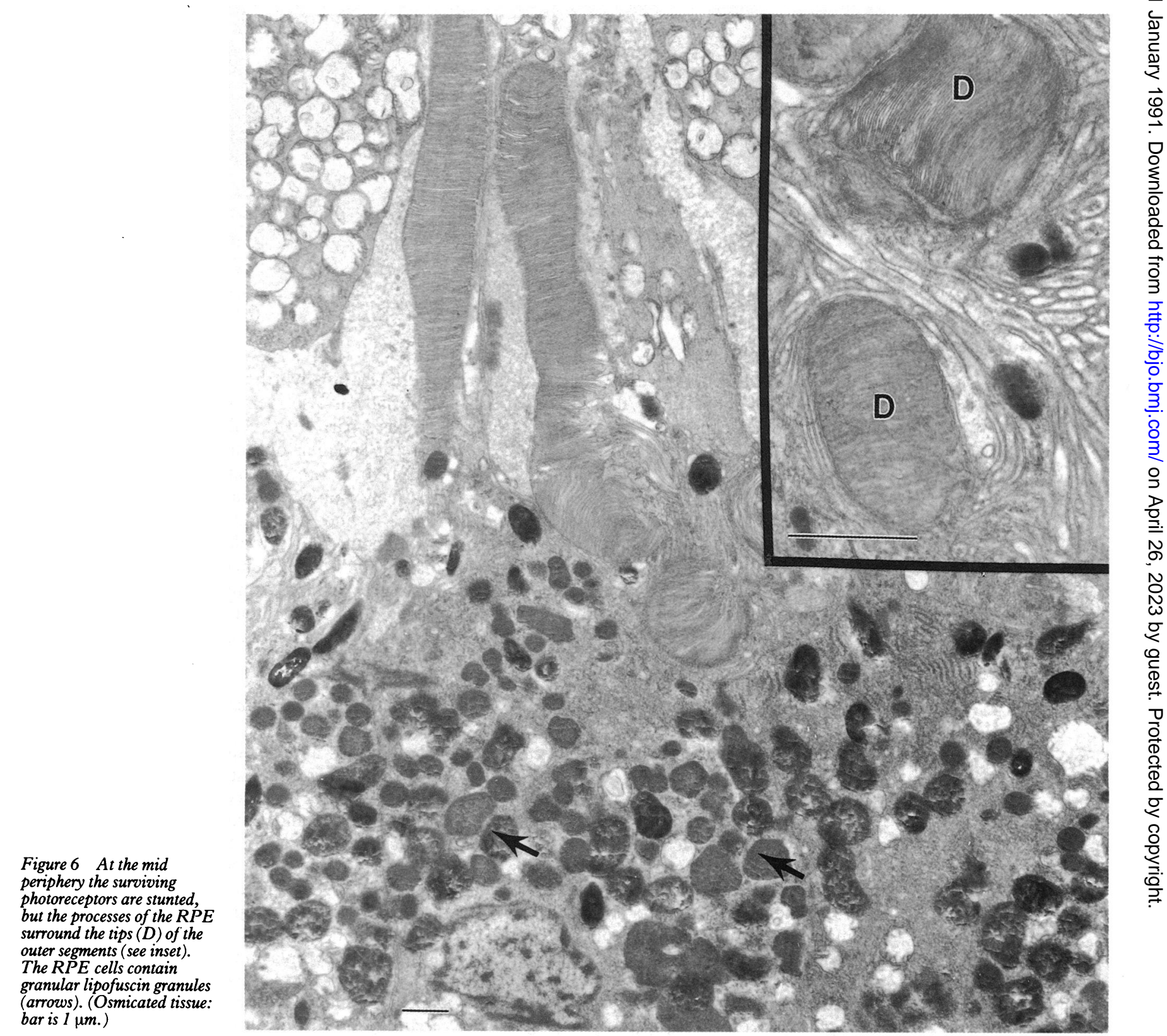


Figure 7 Toward the equator the photoreceptor grating RPE cells are linked by numerous interdigitations

(arrowhead). A macrophage (M) containing

melanophagosomes is present in the gliotic retina. Melanin granules and lipofuscin are sparse within the $R P E$ $(\times 5600$. $)$

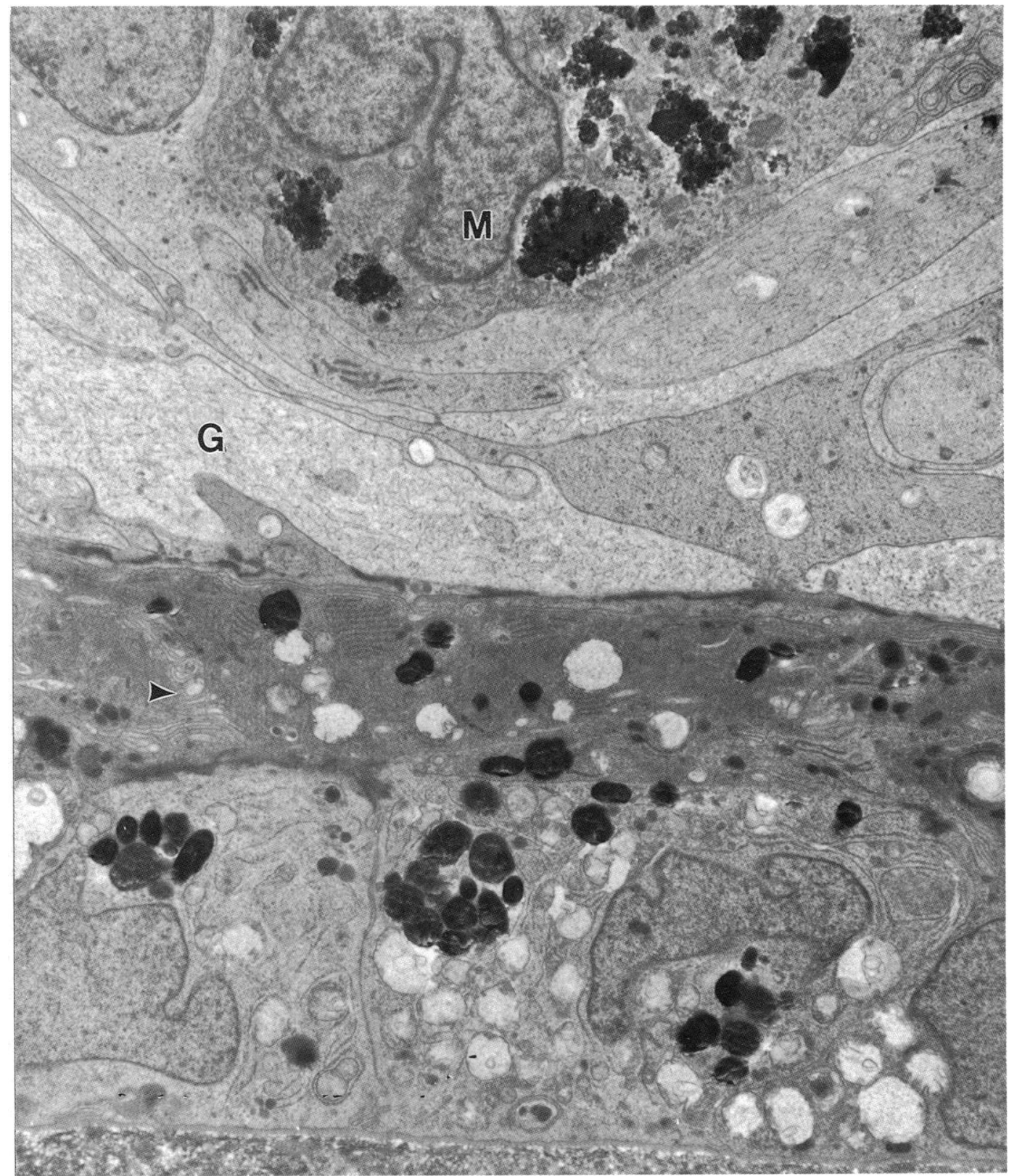

the present case according to current opinion which holds that lipofuscin granules in the RPE represent the degradation products of shed photoreceptor discs, ${ }^{1-7}$ though lipofuscin is also derived from the breakdown of endogenous cytoplasmic lipid-containing membranes such as mitochondrial products. In the normal RPE the number of lipofuscin granules per cell increases from the periphery to the posterior pole, and there is a measurable increase with age throughout the monolayer. ${ }^{461213}$ Because there are no absolute standards for the microscopic measurement of lipofuscin, it is not possible to make direct comparison from our data to previously published work..$^{4613}$ However, our qualitative and quantitative estimates of lipofuscin autofluorescence in control tissue correspond in relative terms with information already available, and this strengthens the data obtained from the traumatised eye.

Our arbitrary value of 50 (SD 25) units for lipofuscin in the RPE of a 19-year-old was about $20 \%$ of that obtained from the 73-year-old eye, namely 250 (SD 50) units, which is not too far from the $50 \%$ difference reported by Wing et $a l^{+}$ in a comparison between 30 years and 80 years. Feeney-Burns et al ${ }^{13}$ gave estimates of 88 granules per cell in the 1-20 age group and 208 granules per cell for the 61-100 age group, that is, about $40 \%$ lower in the younger age group. Accordingly we were encouraged that the mean which there was pathology which could have interfered significantly with the function of the RPE photoreceptor complex did not fall outside the upper and lower limits of the two controls.

The value of $600($ SD 170$)(\mu \mathrm{m})^{2} / 50 \mu \mathrm{m}$ for lipofuscin autofluorescence in a 55-year-old patient with post-traumatic RPE therefore reinforces the light microscopic and electron microscopic findings.

In our search of the literature relevant to RPE hyperlipofuscinosis we gained the impression that the condition was most commonly associated with photoreceptor atrophy and degeneration, which is in contrast to the good preservation of the photoreceptors observed in the case of traumatised eye under discussion. values obtained from eyes $(c)$ and $(d)$ (Table 1 ) in 
Hyperlipofuscinosis in association with photoreceptor degeneration has been described by Weingeist $e t$ al $^{14}$ as a feature of Best's macular dystrophy, but those authors' electron micrographs do not demonstrate a concentration of lipofuscin as excessive as that described in the present case. As case of retinitis pigmentosa (complicated by angle closure glaucoma surgery and aphakia) has been reported by Kolb and Gouras, ${ }^{8}$ and in their case the retinal pigment epithelium appeared to contain large quantities of lipofuscin at the light microscopic level. More recently large quantities of lipofuscin were demonstrated in clusters of RPE cells in a choroideraemia carrier. ${ }^{9}$ The closest approach to the massive accumulation of RPE lipofuscin seen in the present case has been demonstrated in experiments on rats to study the effect of hypovitaminosis $A$ and $E .^{15-17}$ These experiments were designed to interfere with the mechanisms known to be involved in photoreceptor disc lipoprotein maintenance and in protection from peroxidation. Large quantities of lipofuscin accumulate in human ceroid lipofuscinosis ${ }^{10}$ and in animals similarly affected, ${ }^{11} 19$ but the ultrastructural characteristics of ceroid lipofuscin are dissimilar to the lipofuscin seen in normal aging and in the present case.

In contrast to hyperlipofuscinosis described above it was of interest that, when the RPE contained demonstrable iron, lipofuscin was not demonstrable. This was also a feature observed in the siderotic eye used for the present study (Table 1, case d). There was a similar dearth of lipofuscin in the RPE of the present case, in which iron and blood breakdown products were identified at the retinal periphery. The mechanisms by which soluble toxic iron salts interfere with RPE and photoreceptor metabolism are not known. However, at the posterior pole of the present specimen iron was not demonstrable histologically, and, since there was acceptable photoreceptor preservation, siderosis is an unlikely explanation for hyperlipofuscinosis.

It is worth reiterating that our patient has not suffered from familial or acquired systemic disease relevant to the changes in the enucleated eye in the period 1982-90 and his opposite eye has been normal. The pathological findings in the enucleated eye suggest that there was retinal detachment and widespread fragmentation of outer segments at the time of initial injury and that subsequently the retina reattached. The effect of the trauma may have been similar to that described in the pig model for blunt trauma.$^{20} \mathrm{In}$ this model the acute dissolution and fragmentation of photoreceptors is followed by a gradual recovery, with restoration of inner and outer segments. In the present case there was an apparent recovery in the ability of the photoreceptor layer to produce outer segments at the posterior pole, with a failure at the mid periphery and the far periphery.

The RPE lipofuscin content appeared to mirror the status of the photoreceptors, but whether or not the accumulated lipofuscin was a manifestation of a single insult or a persisting low grade dysfunction in degradation remains open to conjecture. The morphology of the accumulated materials within the RPE at the posterior pole was identical to that seen in the aging process $^{361213}$ and differed only in the greatly increased number of the particles. The identification of structures assumed to be photoreceptor phagosomes at various levels in the RPE cells points to some blockage of the degradative system at an early stage. We had anticipated some manifestations of disturbance in RPE exocytosis, which is a feature of normal aging and is recognised by the presence of curvilinear bodies in Bruch's membrane. It was of interest that Bruch's membrane and the choriocapillaris were totally unaffected by the excess of lipofuscin in the RPE monolayer, which contrasts with Best's macular dystrophy. ${ }^{14}$ Even though it is difficult to explain the pathogenesis, it seems justifiable to add this variant of hyperlipofuscinosis to the list of post-traumatic abnormalities which can occur in the human neural retina and RPE.

1 Young RW. Biological renewal. Applications to the eye. Trans Ophthalmol Soc UK 1982; 102: 42-75.

2 Bird AC, Marshall J. Retinal receptor disorders without known metabolic abnormalities. In: Garner A, Klintworth GK, eds. Pathobiology of ocular disease. New York: Dekker, 1982: 1167-220.

3 Feeney L. Lipofuscin and melanin of human retinal pigmen epithelium. Invest Ophthalmol Vis Sci 1978; 17: 583-99.

4 Wing GL, Blanchard GC, Weiter JJ. The topography and age relationship of lipofuscin concentration in the retinal pigment epithelium. Invest Ophthalmol Vis Sci 1978; 17: 601-7.

5 Kitagawa $K$, Nishida S, Ogura $Y$. In vivo quantitation of autofluorescence in human retinal pigment epithelium. autofluorescence in human retinal

6 Weiter JJ, Delori FC, Wing GL, Fitch KA. Retinal pigment epithelial lipofuscin and melanin and choroidal melanin in human eyes. Invest Ophthalmol Vis Sci 1986; 27: 145-52.

7 Pauleikhoff D, Harper A, Marshall J, Bird AC. Aging changes in Bruch's membrane. Ophthalmology 1990; 97: 171-8.

$8 \mathrm{Kolb} \mathrm{H}$, Gouras P. Electron microscopic observations of human retinitis pigmentosa, dominantly inherited. Invest Ophthalmol Vis Sci 1974; 13: 487-98.

9 Flannery JG, Bird AC, Farber DB, Weleber RG, Bok D. A histopathological study of a choroideremia carrier. Invest Ophthalmol Vis Sci 1990; 31: 229-36.

10 Hittner HM, Zeller RS. Ceroid-lipofuscinosis (Batten's disease). Fluorescein angiography electrophysiology, histopathology, ultrastructure, and a review of amaurotic familial idiocy. Arch Ophthalmol 1975; 93: 178-83.

11 Graydon RJ, Jolly RD. Ceroid-lipofuscinosis (Batten's disease): sequential electrophysiologic and pathologic changes in the retina of the ovine model. Invest Ophthalmol Vis Sci 1984; 25: 294-301.

12 Feeney-Burns L, Gao CL, Berman ER. The fate of immunoreactive opsin following phagocytosis by pigment epithelium in human and monkey retinas. Invest Ophthalmol Vis $\mathrm{Sci}$ 1988; 29: 708-19.

13 Feeney-Burns L, Hilderbrand ES, Eldridge S. Aging human RPE: morphometric analysis of macular, equatorial and peripheral cells. Invest Ophthalmol Vis Sci 1984; 25: 195200.

14 Weingeist TA, Kobrin JL, Watzke RC. Histopathology of Best's macular dystrophy. Arch Ophthalmol 1982; 100: $1108-14$

15 Robinson WG, Kuwabara T, Bieri JG. The roles of vitamin E and unsaturated fatty acids in the visual process. Retina 1982; 2: 263-81.

16 Robinson WG, Kuwabara T, Bieri JG. Deficiencies of vitamins $E$ and $A$ in the rat. Retinal damage and lipofuscin accumulation. Invest Ophthalmol Vis Sci 1980; 19: 1030-7.

17 Robinson WG, Kuwabara T, Bieri JG. Vitamin E deficiency and the retina. Photoreceptor and pigment epithelial and the retina. Photoreceptor and pigment ep
changes. Invest Ophthalmol Vis Sci 1979; 7:683-90.

18 Armstrong DA, Siakotos A, Koppang N, Connole E. Studies on the retina and the pigment epithelium in hereditary canine ceroid lipofuscinosis. The distribution of enzymes in the whole retina and pigment epithelium. Invest Ophthalmol Vis Sci 1978; 17: 608-17.

19 Siakotos AN, Armstrong D, Koppang N, Connole E. Studies on the retina and the pigment epithelium in hereditary canine ceroid lipofuscinosis. II. The subcellular distribution of lysosomal hydrolases and other enzymes. Inves Ophthalmol Vis Sci 1978; 17: 618-33.

20 Blight R, Hart JCD. Structural changes in the outer retinal layers following blunt mechanical non-perforating trauma to the globe. An experimental study. BrF Ophthalmol 1977; 61: 573-87. 